\title{
Formación de docentes reflexivos: un enfoque en construcción y disputa
}

Andrea Ruffinelli

\begin{abstract}
Resumen
Revisión bibliográfica en torno a la formación de docentes reflexivos a partir de veinticuatro artículos publicados en revistas arbitradas, entre los años 2009 y 2014. Se desarrolla un breve recuento de la historia del concepto de reflexión y del enfoque del profesor como profesional reflexivo, luego da cuenta de los principales hallazgos, metodologías, argumentos y tensiones presentes en las mediciones, experiencias y documentos analíticos revisados y termina con una discusión que identifica una brecha investigativa en el campo. Los resultados muestran un foco distinto al de la década anterior, optando por la búsqueda de estrategias para el desarrollo de la reflexión, con resultados incipientes, en un marco de creciente rigurosidad científica, y de una progresiva incorporación de nuevas preguntas inspiradas en otras disciplinas, orientadas a develar la forma en que la experiencia podría transformarse en conocimiento mediante la reflexión y la modificación de la práctica.
\end{abstract}

Palabras clave

Reflexión docente - Formación docente - Desarrollo profesional docente - Profesionalización docente.

I- Universidad Alberto Hurtado, 


\title{
Educating reflective teachers: an approach under progress and dispute
}

Andrea Ruffinelli'

\begin{abstract}
Literature review on the training of reflective teachers in twentyfour articles published between 2009 and 2014 in international, specialized and refereed journals. It briefly recounts the history of the concept of reflection and of the approach to teachers as reflective professionals, then it presents the main findings, methodologies, arguments, and tensions that exist in the measurements, experiences and analytical documents reviewed, and discusses a research gap in the field. Findings show a different focus from that of the previous decade, opting for the search for strategies for the development of reflection, with incipient results in the context of increasing scientific rigor, and the gradual incorporation of new questions inspired by other disciplines, which are oriented towards revealing how the experience could be turned into knowledge through reflection and changes in practice.
\end{abstract}

\section{Keywords}

Teacher reflection - Teacher education - Teacher professional development - Teacher professionalization.

I- Universidad Alberto Hurtado, 
En los últimos 25 años la reflexividad se ha constituido en una piedra angular que cruza los idearios formativos de docentes. Esta dimensión del desarrollo profesional representa un eje transversal de la formación inicial docente, erigiéndose como uno de los pilares del perfil del profesor que las instituciones formadoras declaran construir. En este acto, se asume al profesor como un profesional reflexivo, que ha de ser preparado para esta práctica como fuente de aprendizaje profesional permanente (CORNEJO, 2003; GUERRA, 2009; KAASILA; LAURIALA, 2012; RUSSELL, 2005; 2012; ZEICHNER, 1993).

Sin embargo, la literatura da cuenta de un amplio consenso respecto de la ambigüedad del concepto de reflexividad y de la diversidad de sus interpretaciones, caracterizadas por límites difusos que dificultan su definición y falta de acuerdos acerca de cómo enseñarla (ALPER, 2010; CONCHA et al., 2013; FENDLER, 2003; GUERRA, 2009; JAY; JOHNSON, 2002; MARCOS; SÁNCHEZ; TILLEMA, 2011; RUSSELL, 2005; SILVA et al., 2012; THOMPSON; PASCAL, 2012; YAFFE, 2010). Adicionalmente, Russell (2012) alude a la saturación de la presencia del concepto en los programas de formación y a la paradójicamente escasa evidencia de su desarrollo en dichos programas.

En este escenario de disputa parece pertinente revisar el concepto, enfoques, resultados de investigación, tensiones y proyecciones. Este texto recorre la literatura de los últimos cinco años respecto de la formación de docentes reflexivos, discutiendo sobre los avances de la investigación de este campo.

\section{Una aproximación al desarrollo del concepto de reflexión}

En la literatura se pueden identificar tres tradiciones del enfoque del profesor como profesional reflexivo: a) Enseñanza reflexiva (CRUICKSHANK, 1987); b) Práctica reflexiva (SCHÖN, 1983; 2010); y c) Indagación crítica (CORNEJO, 2003; ZEICHNER, 1993). La enseñanza reflexiva (o racionalidad técnica) sostiene que la práctica es profesional cuando se sustenta en evidencia científica, construyendo este aprendizaje mediante una reflexión generada por el análisis de la propia práctica en un contexto de simulación, que ofrece oportunidades de reflexionar a la luz de los hallazgos de la investigación. Desde esta perspectiva, se reconoce un saber reflexivo de base experiencial -a partir de la reflexión acerca de la propia experiencia de práctica docente- que es informado por las teorías existentes (DEWEY, 1989).

La práctica reflexiva concibe la docencia más cercana al arte que a la ciencia, estimando que las ciencias aplicada y técnica son necesarias pero insuficientes para este ejercicio (SCHÖN, 1983). Desde esta perspectiva, la práctica reflexiva alude a un saber que se construye a partir de la experiencia, mediada por una reflexión en y sobre la acción (SCHÖN, 2010), saber que genera nuevo conocimiento práctico capaz de modificar el repertorio del docente, de generar aprendizaje profesional y mejorar con ello la práctica. Esta tradición se distingue de la anterior en la ausencia del componente técnico o de la teoría (SCHÖN, 1998).

Finalmente, la reflexión crítica, sostiene que se investiga y reflexiona con el fin de transformar la práctica, interpelando las condiciones sociales de la experiencia docente, concibiéndola al igual que las dos tradiciones anteriores -como una herramienta al servicio del mejoramiento de la práctica, pero desde un abordaje social y contextual que enfatiza en la transformación (ZEICHNER, 1993).

Considerando los tres enfoques descritos, diversos autores han desarrollado taxonomías de la reflexividad, construyendo categorías sobre las que se ha basado el desarrollo de este campo. Van - Manen (1977) distingue tres niveles jerárquicos de reflexión, homónimos a los enfoques. Zeichner (1993) y Hatton y Smith (1995) se basan en esta clasificación para desarrollar otras similares, sin embargo incorporan un tipo de reflexión de naturaleza descriptiva, ubicándola en un nivel básico. 
Jay y Johnson (2002) se desmarcan de la naturaleza jerárquica de las tipologías planteadas por sus antecesores, proponiendo tres dimensiones de la reflexión: descriptiva, comparativa y crítica, donde la dimensión comparativa se vincula al enfoque de racionalidad técnica, y la dimensión crítica lo hace a su enfoque homónimo. Esta crítica a la naturaleza jerárquica de los niveles es compartida por Russell (2012) argumentando la carencia de evidencia que respalde dicha asunción.

Trascendiendo a estas categorizaciones, la literatura indica que los profesores expertos reflexionan de manera sistemáticamente diferente sobre la enseñanza, en comparación con los profesores principiantes (BERLINER, 1986). Precisa que los expertos razonan sobre situaciones de enseñanza, en tanto los principiantes tienden sólo a describir lo que ven, carecer de conexiones y a no integrar la teoría, en ausencia de principios de la enseñanza y el aprendizaje para fundamentar sus decisiones pedagógicas.

Paralelamente, Fendler (2003) critica la diversidad de interpretaciones de la reflexión pedagógica y de la corriente de reflexividad. Sostiene que los argumentos de apoyo a la reflexividad son tan amplios, generales y divergentes que con frecuencia resultan contradictorios. Se focaliza en la confusión acerca del significado del concepto de reflexión cuando se intenta fundamentarlo simultáneamente en el conocimiento científico (desde una perspectiva más deweyana, reflexión científica) y en la incertidumbre intuitiva (desde una perspectiva schöniana, reflexión artística), combinando arte y ciencia.

Una revisión de la literatura en el campo de las experiencias de desarrollo reflexivo de estudiantes de Pedagogía realizado por Guerra (2009) entre 1998 y 2008 -periodo inmediatamente anterior a la búsqueda desarrollada para este trabajo-, concluye que predominan experiencias con énfasis en la reflexión técnica, aunque débiles en su evaluación, razón por la que releva la falta de evidencia científica que sustente el enfoque reflexivo en la formación inicial docente.
La misma autora define la reflexión como un producto social que requiere mediación o andamiaje de otro y de herramientas que la sitúen, como videos o escritura; señalando la coexistencia de distintos niveles y sugiriendo que su enseñanza requiere ser organizada en torno a preguntas y procesos clave; disponiendo de dos condiciones mínimas para su desarrollo: a) tiempo y espacio para la reflexión; b) formadores preparados para propiciar la reflexión.

\section{Propósito de la revisión}

Esta revisión tiene por finalidad indagar en el concepto de la reflexividad docente, relevando los consensos y argumentos en disputa, considerando resultados de las experiencias diseñadas para el desarrollo de la reflexión en la formación inicial de profesores presentes en la literatura reciente, con miras a la identificación de las actuales líneas investigativas y sus proyecciones. Se enmarca en el trabajo de revisión bibliográfica para el desarrollo de una tesis doctoral en Ciencias de la Educación, y el objetivo se aborda mediante una revisión y sistematización analítica de literatura teórica y empírica en la materia, concluyendo con la identificación de las brechas de conocimiento del campo temático.

\section{Metodología}

La revisión de literatura se ha organizado en base a la sistematización de artículos pesquisados en las bases de datos de la World of Science (ISI y ScIELO) y SCOPUS, usando combinaciones de las siguientes palabras clave: reflect teacher education y pre-service teachers; reflexión docente, reflexividad docente, formación inicial docente. La búsqueda se ha acotado a los últimos cinco años -2009-2014-, considerando que este rango de años cubre el periodo inmediatamente posterior al de la última revisión realizada en Chile acerca del tema (GUERRA, 2009). 
Fueron seleccionados veintidós artículos usando como criterios de inclusión: a) discutir perspectivas teóricas de la reflexividad docente; b) informar resultados de investigaciones empíricas acerca de la reflexividad en estudiantes de pedagogía o profesores principiantes.

A los veintidós artículos se suman dos documentos: un informe de investigación que propone un modelo para el desarrollo reflexivo en la formación inicial docente y un artículo en una revista de otra indexación, considerando a la trayectoria del autor en la materia. Criterio de exclusión fue el que la discusión o experiencia informada se desarrollara en ámbitos distintos a los de la formación inicial docente (otras profesiones o como parte de la formación continua de profesores).

El análisis se realizó bajo una modalidad inductiva, generando categorías que identifican patrones y discrepancias en los marcos conceptuales, métodos y resultados. Los resultados se estructuran en tres categorías: evaluaciones, experiencias y discusiones analíticas, relevando los avances y tensiones del campo investigativo.

\section{Los resultados de la revisión}

Seis documentos dan cuenta de evaluaciones de la reflexividad entre estudiantes de pedagogía; otros doce informan experiencias de desarrollo de la reflexión entre estudiantes o practicantes de pedagogía; mientras seis desarrollan análisis o críticas al concepto o a aspectos del mismo.

\section{Los focos de la literatura reciente: evaluaciones, experiencias y análisis}

\section{Evaluaciones: evidencias en distintos sentidos y la relevancia de la aproximación metodológica}

Seis documentos describen la reflexión pedagógica en estudiantes de carreras pedagógicas, mediante mediciones. El marco conceptual predominante es el de la reflexión crítica, y sólo un trabajo pone el foco en la racionalidad técnica (CONCHA et al., 2013). Las aproximaciones metodológicas se dividen entre cuantitativas y cualitativas, utilizando encuestas en el primer caso, con análisis descriptivo y un número significativo de participantes (ALPER, 2010; GUROL, 2011); o bien producción escrita con análisis inferencial (CONCHA et al., 2013), con menos casos; y estrategias de medición más directas en el caso de la investigación cualitativa, como la producción escrita (CONCHA et al., 2013); memory work, que pone en situación grupal de reflexión desencadenada por recuerdos activados por el investigador mediante preguntas (OVENS; TINNING, 2009); o revisión de productos como portafolios de didáctica de las matemáticas (KAASILA; LAURIALA, 2012; ZHU, 2011).

En dos trabajos -utilizando encuestas a estudiantes de sólo una institución- se reportan evidencias de pensamiento reflexivo como un rasgo cotidiano entre los docentes en formación (ALPER, 2010; GUROL, 2011) , mientras en las cuatro restantes -de medición más directase reporta la presencia de bajos niveles de reflexividad (CONCHA et al., 2013; KAASILA; LAURIALA, 2012; OVENS; TINNING, 2009; ZHU, 2011). Concha y otros autores (2013) miden reflexividad en 82 estudiantes de pedagogía en educación básica y parvularia de último año de tres universidades chilenas, y dan cuenta del predominio de reflexiones desde el sentido común, y no desde el conocimiento teórico.

Los otros tres documentos que reportan mediciones muestran hallazgos basados en las categorías propuestas por Jay y Johnson (2002) y Van-Manen (1977), y observan desempeños reflexivos según contextos de reflexión. Ovens y Tinning (2009) y Zhu (2011) dan cuenta de muy baja presencia de reflexión crítica, observándose más bien un nivel descriptivo. Zhu (2011) concluye que los distintos contextos de la práctica generan distintos tipos de reflexión, pero en general vinculados a una racionalidad técnica sobre la acción. En una línea similar, Kaasila y Lauriala (2012) -mediante el análisis de 53 portafolios- 
Tabla 1 - Los documentos revisados

\begin{tabular}{|c|c|c|c|c|c|}
\hline Autor(es) & Año & Título artículo & Revista & Lugar & Indexación \\
\hline \multicolumn{6}{|l|}{ Evaluaciones } \\
\hline Alper, A. & 2010 & Critical Thinking Disposition of Pre-Service Teachers & $\begin{array}{l}\text { Egitim Ve Bilim-Education } \\
\quad \text { and Science }\end{array}$ & Turquía & ISI \\
\hline $\begin{array}{l}\text { Concha, S.; Hernández, } \\
\text { C.; del Río, F.; Romo, F. y } \\
\text { Andrade, L. }\end{array}$ & 2013 & $\begin{array}{c}\text { Reflexión pedagógica en base a casos y lenguaje académico } \\
\text { en estudiantes de último año de Pedagogía en Educación } \\
\text { Básica }\end{array}$ & Calidad en la Educación & Chile & ScIELO \\
\hline Gurol, A. & 2011 & $\begin{array}{l}\text { Determining the reflective thinking skills of pre-service } \\
\text { teachers in learning and teaching process }\end{array}$ & $\begin{array}{l}\text { Egitim Ve Bilim-Education } \\
\quad \text { and Science }\end{array}$ & Turquía & ISI \\
\hline Kaasila, R., Lauriala, A. & 2012 & $\begin{array}{l}\text { How do pre-service teachers' reflective processes differ in } \\
\text { relation to different contexts? }\end{array}$ & $\begin{array}{l}\text { European Journal of Teacher } \\
\text { Education }\end{array}$ & Finlandia : & $|S|$ \\
\hline Ovens, A., Tinning, R. & 2009 & $\begin{array}{c}\text { Reflection as situated practice: A memory-work study of lived } \\
\text { experience in teacher education }\end{array}$ & $\begin{array}{l}\text { Teaching and Teacher } \\
\text { Education }\end{array}$ & $\begin{array}{l}\text { N. } \\
\text { Zelanda }\end{array}$ & SCOPUS \\
\hline Zhu, X. & 2011 & $\begin{array}{l}\text { Student teachers' reflection during practicum: plenty on } \\
\text { action, few in action }\end{array}$ & Reflective practice & EEUU & SCOPUS \\
\hline \multicolumn{6}{|c|}{ Experiencias } \\
\hline Frick, L., Carl, A., Beets, P. & 2010 & $\begin{array}{l}\text { Reflection as learning about the self in context: mentoring as } \\
\text { catalyst for reflective development in pre-service teachers }\end{array}$ & $\begin{array}{l}\text { South African Journal of } \\
\text { Education }\end{array}$ & Sudáfrica & $|S|$ \\
\hline Gelfuso, A., Dennis, D. V & 2014 & $\begin{array}{l}\text { Getting reflection off the page: The challenges of developing } \\
\text { support structures for pre-service teacher reflection }\end{array}$ & $\begin{array}{l}\text { Teaching and Teacher } \\
\text { Education }\end{array}$ & EEUU & $|S|$ \\
\hline Yaffe, E. & 2010 & $\begin{array}{l}\text { The reflective begginer: using Theory and practice to facilitate } \\
\text { reflection among newly qualified teachers }\end{array}$ & Reflective Practice & Israel & SCOPUS \\
\hline $\begin{array}{l}\text { Silva, A.; Rubio, G.; Herrera, } \\
\text { V.; Nervi, H. }\end{array}$ & 2012 & $\begin{array}{l}\text { Modelo para el desarrollo de capacidades reflexivas y } \\
\text { didácticas en estudiantes de educación parvularia y básica } \\
\text { durante la práctica profesional. FONIDE F-611145 }\end{array}$ & - & Chile & $\begin{array}{l}\text { Informe de } \\
\text { investigación }\end{array}$ \\
\hline Chung, H. Q., van Es, E. A. & 2014 & $\begin{array}{c}\text { Pre-service teachers' use of tools to systematically analyze } \\
\text { teaching and learning }\end{array}$ & $\begin{array}{l}\text { Teachers and Teaching: } \\
\text { theory and practice }\end{array}$ & EEUU & SCOPUS \\
\hline $\begin{array}{l}\text { Bates, A.; Ramírez, L. \& } \\
\text { Drits, D. }\end{array}$ & 2009 & $\begin{array}{c}\text { Connecting university supervision and critical reflection: } \\
\text { mentoring and modeling }\end{array}$ & Irish Educational Studies & EEUU & $|S|$ \\
\hline $\begin{array}{l}\text { Oakley, G., Pegrum, M., } \\
\text { Johnston, S. }\end{array}$ & 2014 & $\begin{array}{l}\text { Introducing e-portfolios to pre-service teachers as tools for } \\
\text { reflection and growth: lessons learnt }\end{array}$ & $\begin{array}{l}\text { Asia-Pacific Journal of } \\
\text { Teacher Education }\end{array}$ & Australia : & $|S|$ \\
\hline $\begin{array}{l}\text { Blomberg, G., Sherin, M. G., } \\
\text { Renkl, A., Glogger, I., Seidel, T. }\end{array}$ & 2014 & $\begin{array}{l}\text { Understanding video as a tool for teacher education: } \\
\text { investigating instructional strategies to promote reflection }\end{array}$ & Instructional Science & Alemania : & SCOPUS \\
\hline Mellita, J., Ryan, J. & 2014 & $\begin{array}{l}\text { Learning in the practicum: engaging pre-service teachers in } \\
\text { reflective practice in the online space }\end{array}$ & $\begin{array}{l}\text { Asia-Pacific Journal of } \\
\text { Teacher Education }\end{array}$ & Australia : & ISI \\
\hline $\begin{array}{l}\text { Moore-Russo, D. A., Wilsey, } \\
\text { J. N. }\end{array}$ & 2014 & $\begin{array}{l}\text { Delving into the meaning of productive reflection: A study of } \\
\text { future teachers' reflections on representations of teaching }\end{array}$ & $\begin{array}{l}\text { Teaching and Teacher } \\
\text { Education }\end{array}$ & EEUU & $|S|$ \\
\hline Daniel, et al. & 2013 & $\begin{array}{l}\text { Collaborative feedback and reflection for professional } \\
\text { growth: preparing first-year pre-service teachers for } \\
\text { participation in the community of practice }\end{array}$ & $\begin{array}{l}\text { Asia-Pacific Journal of } \\
\text { Teacher Education }\end{array}$ & Australia : & $|S|$ \\
\hline Sims, L., Walsh, D. & 2009 & $\begin{array}{l}\text { Lesson Study with preservice teachers: Lessons from } \\
\text { lessons }\end{array}$ & $\begin{array}{l}\text { Teaching and Teacher } \\
\text { Education }\end{array}$ & EEUU & SCOPUS \\
\hline \multicolumn{6}{|c|}{ Análisis } \\
\hline $\begin{array}{l}\text { Marcos, J. M., Sanchez, E., } \\
\text { Tillema, H. H. }\end{array}$ & 2011 & Promoting teacher reflection: what is said to be done & $\begin{array}{l}\text { Journal of Education for } \\
\text { Teaching }\end{array}$ & España & $|S|$ \\
\hline Russell, T. & 2012 & $\begin{array}{c}\text { Cambios paradigmáticos en la formación de profesores: } \\
\text { Peligros, trampas y la promesa no cumplida del profesional } \\
\text { reflexivo }\end{array}$ & Encounters on Education & Canadá & $\begin{array}{l}\text { Dialnet, } \\
\text { Latindex }\end{array}$ \\
\hline $\begin{array}{l}\text { Holloway, S. M., Gouthro, } \\
\text { P. A. }\end{array}$ & 2011 & Teaching resistant novice educators to be critically reflective & $\begin{array}{l}\text { Discourse-Studies in } \\
\text { the Cultural Politics of } \\
\text { Education }\end{array}$ & Canadá & $|S|$ \\
\hline Ross, J. & 2014 & $\begin{array}{l}\text { Performing the reflective self: audience awareness in high- } \\
\text { stakes reflection }\end{array}$ & Studies in Higher Education & $\begin{array}{l}\text { Reino } \\
\text { Unido }\end{array}$ & $|S|$ \\
\hline Thompson, N., Pascal, J. & 2012 & Developing critically reflective practice & Reflective Practice & Australia & SCOPUS \\
\hline Zink, R., \& Dyson, M. & 2009 & $\begin{array}{l}\text { What does it mean when they don't seem to learn from } \\
\text { experience? }\end{array}$ & $\begin{array}{l}\text { Cambridge Journal of } \\
\text { Education }\end{array}$ & Australia & $|S|$ \\
\hline
\end{tabular}

Fuente: Elaboración propia. 
concluyen que varía la naturaleza y contenido de la reflexión dependiendo del contexto; y que la lectura de investigación científica genera mayor profundidad reflexiva cuando se evalúa en contexto teórico.

En síntesis, la evidencia sugiere que la aproximación metodológica incide sobre los hallazgos. El autorreporte parece ser menos apropiado, dada la deseabilidad en la respuesta, en comparación con la evaluación más directa.

Finalmente, los marcos conceptuales desde los que se plantean estas mediciones coinciden en asumir la reflexión crítica y transformadora y como el nivel sofisticado al que aspirar, desde una concepción que contiene la racionalidad técnica, es decir, requiriendo del manejo de conocimientos a la luz de los cuales reflexionar.

\section{Experiencias de promoción de la reflexividad docente}

Doce experiencias en siete países indagaron acerca del rol de la tutoría/andamiaje en el desarrollo reflexivo, desde abordajes metodológicos preferentemente cualitativos.

Las experiencias reportan mayoritariamente- iniciativas puntuales, con un pequeño número de participantes, en una única institución formadora. Las excepciones corresponden a cursos del proceso formativo, generalmente impartidos por los investigadores. La mitad de estas experiencias se enmarcan en la reflexividad crítica como nivel a aspirar (BATES; RAMÍREZ; DRITS, 2009; DANIEL; AUHL; HASTINGS, 2013; FRICK; CARL; BEETS, 2010; MOORE-RUSSO; WILSEY, 2014; SILVA et al., 2012; YAFFE, 2010), incluyendo en este nivel la racionalidad técnica necesaria para alcanzarla.

Estas experiencias indagan en el rol del tutor en la estructuración, mediación o andamiaje de la experiencia reflexiva, basadas en planteamientos vigostkianos de aprendizaje social, y/o feuersteniananos sobre mediación, u otros (CHUNG; VAN ES, 2014; DANIEL; AUHL; HASTINGS, 2013; GELFUSO; DENNIS, 2014; JONES; RYAN, 2014; MOORE-
RUSSO; WILSEY, 2014; OAKLEY; PEGRUM; JOHNSTON, 2014; SILVA et al., 2012; SIMS; WALSH, 2009; YAFFE, 2010).

Nueve de las doce experiencias apuestan en la centralidad del rol estructurador del formador, ya sea como experto en pedagogía o didáctica, en reflexión, o ambos (BATES; RAMÍREZ; DRITS, 2009; CHUNG; VAN ES, 2014; DANIEL; AUHL; HASTINGS, 2013; FRICK; CARL; BEETS, 2010; GELFUSO; DENNIS, 2014; OAKLEY; PEGRUM; JOHNSTON, 2014; SILVA et al., 2012; SIMS; WALSH, 2009; YAFFE, 2010); mientras dos experiencias comparan los efectos del andamiaje o no andamiaje en la práctica reflexiva (BLOMBERG et al., 2014; JONES; RYAN, 2014); y una experiencia analiza los efectos de la promoción de la reflexión sin andamiaje del tutor (MOORE-RUSSO; WILSEY, 2014).

\section{Andamiaje versus no andamiaje: tensionando el rol del tutor}

Cuatro de las doce experiencias comparan los efectos de la presencia o ausencia de andamiaje en la práctica reflexiva (BATES; RAMÍREZ; DRITS, 2009; BLOMBERG et al., 2014; JONES; RYAN, 2014; MOORERUSSO; WILSEY, 2014), arribando a resultados inconsistentes entre sí.

Dos estudios arrojan resultados internamente consistentes, desde aproximaciones metodológicas distintas, pero en sentidos opuestos (BLOMBERG et al., 2014; JONES; RYAN, 2014). En tanto, otra experiencia verifica los efectos de la promoción de la reflexión sin andamiaje del tutor (Moore-Russo y Wilsey, 2014), reportando efectos similares a los obtenidos con andamiaje.

Blomberg y otros autores (2014) experimentan cómo diferentes niveles de estructuración de la reflexión inducen diferentes patrones, en dos cursos mediante análisis de videos en diarios reflexivos individuales. Usando análisis estadístico multinivel de los resultados hacen un hallazgo que podría ser clave para la discusión acerca de las decisiones a tomar según las necesidades pedagógicas de la situación 
formativa: reportan que al ofrecer estructura se obtienen resultados rápidos, aunque no persisten en el tiempo, en cambio, cuando la reflexión se promueve libremente, el resultado es una reflexión más profunda y persistente en el tiempo, aunque de más lento desarrollo.

Por su parte, Jones y Ryan (2014) analizan la discusión online grupal, con y sin estructura, al interior de un curso. Reportan que en ninguno de los dos grupos la reflexión llega a ser crítica ni a incorporar los elementos de la teoría, concluyendo que ni la oportunidad abierta de reflexión ni la oportunidad estructurada son suficientes para gatillar reflexión crítica.

Como contrapunto, Moore-Russo y Wilsey (2014) analizan cualitativamente los efectos de la ausencia de andamiaje explícito de un tutor en el desarrollo reflexivo mediante la exposición de estudiantes de último año de Pedagogía a animaciones de clases de álgebra sobre las que deben reflexionar en un foro electrónico asincrónico grupal, libremente. Concluyen que estrategias de enseñanza como las animaciones o videos necesitarian menos andamios y serían beneficiosos, pues la reflexión derivada de ellos es similar a la de docentes experimentados, estableciendo comparaciones entre prácticas pedagógicas y formulando críticas, sin requerir instrucciones para centrarse en la enseñanza, ni para considerar otras perspectivas o integrar los diferentes aspectos de la enseñanza. Sin embargo, reportan que el éxito no fue transversal al momento de hacer conexiones entre la investigación, la teoría y la práctica.

Otro estudio analiza desde la perspectiva de los tutores el rol de la tutoría y modelaje en el desarrollo de la reflexividad (BATES; RAMÍREZ; DRITS, 2009), concluyendo que se trata de un proceso no natural que requiere ser modelado y muy estructurado.

En síntesis, algunos estudios señalan que la interacción con tutores expertos en Pedagogía es insuficiente para alcanzar niveles altos de reflexión, siendo muy escasa la reflexión crítica o la integración de la teoría (DANIEL; AUHL; HASTINGS, 2013; GELFUSO; DENNIS, 2014;
JONES; RYAN, 2014; OAKLEY; PEGRUM; JOHNSTON, 2014); y cuando se compara la reflexión con o sin andamiaje rara vez se encontró reflexión crítica en ambos casos, aunque los propios autores sugieren que un sesgo importante puede haber sido introducido por la falta de experticia y/o compromiso de los tutores (DANIEL; AUHL; HASTINGS, 2013; JONES; RYAN, 2014; OAKLEY; PEGRUM; JOHNSTON, 2014).

\section{Andamiaje individual}

Tres experiencias aportan evidencia respecto del andamiaje o tutoría individual: una con resultados robustos a favor (YAFFE, 2010), otra con resultados menos consistentes (FRICK; CARL; BEETS, 2010), y otra con resultados desfavorables (OAKLEY; PEGRUM; JOHNSTON, 2014). Yaffe (2010), a partir de una investigación acción de trabajo individual, tutor/estudiante, y usando videogafas -dispositivo que permite filmar la clase desde la perspectiva del profesorconcluye que los estudiantes desarrollan nuevas percepciones y perspectivas pedagógicas, alcanzando niveles de problematización de la docencia que nuevas estrategias, desarrollando conciencia de su acción.

Por su parte, Frick y otros autores (2010) analizaron el efecto de la mediación de un tutor en el desarrollo de distintos niveles de reflexión, durante la última práctica profesional. Usando entrevistas concluyen que la tutoría individual tiene más efectos en el nivel jerárquico de misión (reflexión de nivel medio, relativo al estudiante en relación con otros, al sentido de pertenencia a algo más grande, vinculado al conocimiento profesional).

Finalmente, Oakley, Pegrum y Johnston (2014) evaluaron los efectos de una tutoría individual mediante escritura en portafolios, reportando que tras la intervención la mayoría alcanzaba niveles bajos o medios de reflexión, aunque también previenen respecto del efecto que pudo haber tenido un insuficiente compromiso y visión compartida de la tarea por parte de los formadores. 


\section{Andamiaje grupal}

Cinco experiencias reportan evidencias respecto de la mediación grupal para el desarrollo reflexivo. Una de ellas con resultados positivos robustos (CHUNG; VAN ES, 2014), tres con resultados menos concluyentes (DANIEL; AUHL; HASTINGS, 2013; SILVA et al., 2012; SIMS; WALSH, 2009) y uno con resultados desfavorables para esta alternativa (GELFUSO; DENNIS, 2014).

Chung y Van Es (2014), mediante análisis de portafolios y videos en un curso para certificar profesores, concluyen que ofrecer andamiaje hace que los estudiantes usen estrategias reflexivas de docentes expertos. En tanto, Silva y otros autores (2012) desarrollan un cuasi-experimento de talleres de simulación para construcción colaborativa de la reflexión. Concluyen que favorecen el desarrollo de la capacidad reflexiva, más en las dimensiones descriptiva y dialógica y menos en la dimensión transformativa, sin embargo, no se informa de una línea base que permita comprender la envergadura de este hallazgo.

En una línea similar, Sims y Walsh (2009) implementaron simulaciones clínicas en un curso los dos primeros años de la formación, en un autoestudio de la adaptación de la lesson study (experiencia auténtica de análisis colaborativo del video real de una clase). Concluyen que puede ayudar a mejorar la reflexión y la práctica en el largo plazo, observando mejores resultados cuando el tutor asume un rol muy estructurador.

Como contrapunto, Gelfuso y Dennis (2014) mediante un experimento formativo tensionan la teoría deweyana de reflexividad -fundamentada en la integración de la teoría a la reflexión- a fin de determinar su utilidad en la práctica. Usando filmaciones, un formador modela y guía muy estructuradamente hacia decisiones justificadas en la práctica. Los resultados indican que fue difícil la reflexión colaborativa y que la reflexión informada por la teoría no ocurrió, concluyendo que las conversaciones con otro conocedor son insuficientes.

Finalmente, y en una línea similar, Daniel, Auhl y Hastings (2013) implementan durante un semestre de la formación una experiencia de reflexión colaborativa entre pares mediante modelaje y alta estructura. También reportaron dificultades para desarrollar reflexión de manera colaborativa, así como progresiva conciencia del valor de dicha experiencia.

En síntesis, experiencias que arrojan evidencia inconsistente respecto de que la mediación más o menos estructurada de un tutor, individual o grupal, favorezca sustantivamente el desarrollo reflexivo de profesores en formación.

\section{Documentos analíticos}

Por último, seis de los veinticuatro documentos revisados discuten el enfoque del profesor como profesional reflexivo. Cuatro de estos seis artículos relevan elementos que observan ausentes y necesarios de incorporar. Marcos, Sánchez y Tillema (2011) argumentan falta de sustento teórico del enfoque desde un meta-análisis sobre 122 artículos españoles -publicados entre 1985 y 2005- que reportaban experiencias sobre reflexividad con estudiantes de Pedagogía. Destacan la ausencia de fundamentos sociológicos $\mathrm{y}$ de acuerdo acerca del concepto y de cómo desarrollar la reflexividad. Asimismo, señalan que generalmente se abordan sus atributos, más que cómo desarrollarlos.

Thompson y Pascal (2012) comparten esos argumentos, aluden también al escaso desarrollo de la teoría de la práctica reflexiva, en oposición a una literatura amplia que informa experiencias, y junto a Holloway y Gouthro (2011) hacen una crítica a la neutralidad sociopolítica del enfoque, planteando que el neoliberalismo imperante atenta contra el desarrollo reflexivo, alejando de la toma de consciencia de su rol en la sociedad y del carácter político y social de su experiencia. 
En palabras de Thompson y Pascal (2012), el problema de la falta de tiempo (al que alude Guerra en su revisión del año 2009) no es tanto la falta de tiempo por sí mismo, sino una cultura que no prioriza la reflexión. Identifica al management o gerencialismo como amenaza para la práctica reflexiva, en la medida en que el énfasis en el control de gestión por sobre la autonomía profesional genera hostilidad hacia la práctica reflexiva, e hipotetiza que el auge del enfoque de la práctica reflexiva puede ser una reacción contra el gerencialismo, contra la insatisfacción generada por la desconfianza y la pérdida de respeto profesional, relevando el peligro que implica la persistencia de un enfoque acrítico en estas condiciones.

Siempre en la línea de la dinámica del poder, Ross (2014) argumenta en contra de la calificación en la evaluación del desarrollo reflexivo de los estudiantes, toda vez que la reflexión de los estudiantes sería producto de una acción estratégica para lograr buenas calificaciones, en una mirada a la práctica reflexiva desde las estructuras de poder.

Los dos trabajos restantes también cuestionan, ya sea el supuesto básico del enfoque o los resultados de su implementación. Zink y Dyson (2009) cuestionan el aprendizaje profesional derivado de la experiencia. Evocan a Dewey al señalar que la interacción entre la experiencia y la reflexión genera aprendizaje y significado, constituyéndose la reflexión en el puente entre experiencia y aprendizaje, permitiendo que la experiencia transforme en conocimiento reconocible, señalando que la experiencia es condición necesaria pero no suficiente para el aprendizaje. También relevan el carácter social de la experiencia, señalando que el contexto y las relaciones entre los participantes y el contexto son fundamentales en la determinación de lo que es posible aprender.

Finalmente, Russell (2012) cuestiona los resultados de la implementación del enfoque, concluyendo que no se ha logrado el objetivo de formar profesores reflexivos y construye una hipótesis para explicarlo, relativa a la persistencia de las prácticas pedagógicas tradicionales, que se detalla más adelante. El autor revisa una selección de seis ejemplos de literatura especializada representativos de distintos enfoques sobre reflexión docente, tras lo que sostiene que la promesa del profesional reflexivo ha sido incumplida. Señala que se dispone de escasos ejemplos de iniciativas en que los profesores principiantes logran aprender de su experiencia personal, junto a la guía de un formador, a la vez que refuerza la idea de la necesidad de ayudar a los profesores en formación a aprender de sus experiencias, y que ello requiere de un modelado explícito y deliberado, pero que en su lugar se ha instalado sólo un cambio discursivo, que no ha afectado la forma de enseñar, debido a la complejidad que implican los cambios paradigmáticos, y la estabilidad de las prácticas docentes.

En síntesis, la emergencia de una literatura que levanta factores nuevos, vinculados a las dinámicas del poder en sociedades regidas por sistemas neoliberales $\mathrm{y}$ por una estabilidad paradigmática $\mathrm{y}$ de prácticas que constituirían barreras estructurales reñidas con la práctica reflexiva.

\section{Discusión}

La investigación se organiza fundamentalmente en torno a experiencias, discusiones analíticas y críticas acerca de la teoría de la práctica reflexiva y mediciones de la reflexividad, asumiendo énfasis distintos a los encontrados en la década anterior. Se ha transitado del énfasis en la pasión, lógica y convicción en la configuración y consolidación del enfoque -que logró trascender las agendas políticas y las declaraciones de los programas formativos, pese a la insuficiente evidencia de sustento que se expresó en el establecimiento de taxonomías y experiencias vinculadas a ellas, y particularmente a experiencias sustentadas en la racionalidad técnica, orientadas a develar la forma en que se estimula el desarrollo del pensamiento reflexivo- hacia un avance en la 
construcción científica de la teoría del enfoque del profesor como profesional reflexivo, con resultados todavía incipientes, surgimiento de tensiones -como el de la opción por una mayor o menor estructuración/andamiaje de la experiencia reflexiva- y aparición de nuevas preocupaciones, como la de la incorporación -todavía a nivel de reflexión teórica- de la dimensión sociopolítica a la teoría del enfoque.

$\mathrm{Si}$ bien es cierto que los hallazgos recientes no suelen dar cuenta de implicancias socio políticas e ideológicas de la práctica reflexiva que trasciendan a la identificación del nivel crítico de reflexión, resulta especialmente interesante el desarrollo incipiente de una línea de literatura analítica que incorpora estas categorías a la discusión, permitiendo dar cuenta de una dimensión del enfoque todavía muy poco presente en la investigación empírica.

La revisión de la literatura reciente arroja evidencia en sentidos opuestos para las mediciones de la reflexividad de estudiantes de Pedagogía, y una esperable tendencia a evidencia más positiva respecto de las capacidades reflexivas docentes cuando la aproximación metodológica es autorreporte.

Respecto de las experiencias, la literatura, al igual que en el periodo anterior (Guerra, 2009), sigue refiriendo dificultad para identificar modelos sistemáticos efectivos para desarrollar la reflexión pedagógica en los futuros docentes, dando cuenta de un nivel de desarrollo del campo investigativo todavía incipiente. Predominan las investigaciones de restringido alcance, habitualmente desarrolladas con estudiantes de una sola institución formadora, en periodos acotados, usualmente el transcurso de una práctica o mediciones transversales en un momento determinado, o bien intervenciones aisladas, en el marco de un curso puntual, o de una práctica determinada.

El foco está puesto en discernir acerca de la efectividad de opciones con mayor o menor andamiaje por parte de los formadores, mediante experiencias apoyadas o bien sustentadas en herramientas como videos, animaciones, discusiones, portafolios, entre otras. Algunas investigaciones experimentales y algunas de naturaleza cualitativa dan cuenta de resultados consistentes de intervenciones orientadas al desarrollo de prácticas reflexivas, mediadas por un tutor experimentado en conocimientos pedagógicos y en práctica reflexiva, que hacen uso de algunos dispositivos facilitadores de la experiencia reflexiva, como la filmación y discusión de videos, videogafas, bitácoras, conversaciones grupales o individuales con el tutor. En general, se releva el vínculo de la reflexión con el análisis a la luz de saberes pedagógicos, aunque también se observan experiencias que no aluden necesariamente a ello.

Y un punto crítico: escasos los estudios que reportan lo que ocurre con las habilidades reflexivas en el tiempo, tras finalizar la experiencia que la promueve, aunque se dispone de algunos artículos en tal sentido (CHUNG; VAN ES, 2014), aunque no es posible hacer generalizaciones a partir de este estudio, dado el contexto de certificación en que fue desarrollado, lo que refuerza la necesidad de profundizar en este tipo de indagaciones.

\section{Lo que se sabe}

Los hallazgos comparados de las mediciones y experiencias sugieren la presencia de bajos niveles de reflexividad entre profesores en formación, reflexión que tienden a alcanzar no más allá de una forma descriptiva, con bajo nivel de integración de los conocimientos promovidos en la formación. Desde esta perspectiva, y en la línea de lo planteado por Dewey (1989) respecto a la necesidad de que la base experiencial del conocimiento profesional sea informada por las teorías existentes, se observa en la literatura una perspectiva que releva esta necesidad de contar con una base sólida de conocimiento que debe estar disponible para la reflexión (CONCHA et al., 2013; DARLING-HAMMOND; BRANSFORD, 2005; KAASILA; LAURIALA, 2012; MARCOS; SÁNCHEZ; TILLEMA, 2011; SCHÖN, 1983; YAFFE, 2010; YOUNGS; BIRD, 2010). 


\section{Lo que requiere seguir siendo investigado}

La literatura revisada da cuenta de evidencia favorable (BATES; RAMÍREZ; DRITS, 2009; CHUNG; VAN ES, 2014; YAFFE, 2010) como desfavorable (GELFUSO; DENNIS, 2014; JONES; RYAN, 2014; MOORE-RUSSO; WILSEY, 2014; OAKLEY; PEGRUM; JOHNSTON, 2014) respecto del rol del tutor/formador en el desarrollo reflexivo, y del andamiaje o necesidad de estructurar la experiencia reflexiva.

Entre los estudios que tensionan el rol estructurador del tutor y entre los que complementan dicho rol con otras herramientas que ayuden al desarrollo del pensamiento reflexivo, se observa el uso de herramientas que son identificadas como portadoras de potencial andamiaje intrínseco para el desarrollo reflexivo, como el análisis de videos (BLOMBERG et al., 2014; CHUNG; VAN ES, 2014), las videogafas (YAFFE, 2010), o las animaciones (MOORE-RUSSO; WILSEY, 2014). Algunos estudios que las incluyeron proveen evidencias a favor de su uso para el desarrollo reflexivo, sin embargo, estas experiencias no evaluaron estas modalidades en diseños controlados; mientras que otras experiencias que las incluyeron no obtuvieron resultados particularmente favorables a su uso (GELFUSO; DENNIS, 2014), Sin embargo, es importante considerar que un grupo de estudios que aporta evidencia desfavorable al andamiaje (DANIEL; AUHL; HASTINGS, 2013; GELFUSO; DENNIS, 2014; OAKLEY; PEGRUM; JONSTON, 2014), reconocen en su diseño debilidades de validez interna, en el control de la intervención.

Resultan particularmente interesantes para el diseño de procesos formativos los hallazgos de Blomberg y otros autores (2014) a favor del andamiaje altamente estructurado cuando el requerimiento pedagógico son resultados rápidos, advirtiendo de la menor persistencia y profundidad de estos resultados en comparación a la persistencia y profundidad alcanzada sin estructura, pero en mayor tiempo.
Otro aspecto en que los resultados de investigación no son todavía concluyentes es la efectividad del tipo de tutoría, individual o grupal, para el logro del desarrollo reflexivo. Algunos estudios aportan evidencia a favor de la tutoría individual (FRICK; CARL; BEETS, 2010; YAFFE, 2010), mientras otros lo hacen respecto de la construcción colaborativa de la reflexión, incluyendo a los pares (DANIEL; AUHL; HASTINGS, 2013; SILVA et al., 2012; SIMS; WALSH, 2009).

Una situación similar se observa respecto de la efectividad de la estrategia de simulaciones clínicas en el desarrollo de la reflexividad (SILVA et al., 2012; SIMS; WALSH, 2009) y sus adaptaciones desde las lesson study, el uso de los portafolios (CHUNG; VAN ES, 2014).

En general, las razones para la no conclusividad o para la ausencia de resultados favorables parecen ligadas fundamentalmente a dos factores: a) sesgos de validez interna de los estudios atribuibles a la falta de control sobre la intervención, relativas a limitaciones de los propios tutores para conducir procesos reflexivos, en términos de saberes pedagógicos, saberes reflexivos, de acompañamiento y/o compromiso con la actividad; y b) escasa consideración de variables o factores críticos, como, por ejemplo, la necesidad de coherencia sistémica orientada a desarrollar la capacidad reflexiva transversalmente durante el proceso formativo; o el hecho de que los profesores en formación requieren adquirir más experiencia en la práctica y en la reflexión, para alcanzar un desarrollo reflexivo experto, así como requieren desarrollar mayor conocimiento pedagógico a la luz del que reflexionar sobre su práctica, o de la influencia de factores sociopolíticos actuando como barreras estructurales para la reflexión.

\section{Conclusiones que identifican una brecha}

La literatura destinó más de veinte años a una discusión teórica prescriptiva a partir de reflexiones de autores influyentes, sin sustento en la evidencia científica, y si bien es cierto que 
esta forma de desarrollo del enfoque persiste en un sector de la investigación, y particularmente de la experiencia formativa, es posible identificar un viraje hacia la construcción teórica del campo a partir de la evidencia empírica en los últimos cinco años, con evidencias todavía incipientes respecto a los modos en que se logra alcanzar formas de pensamiento reflexivo a nivel crítico o transformador, y escaso abordaje respecto de las maneras en que la reflexión sobre la experiencia se convierte en conocimiento profesional y se traduce en mejores prácticas pedagógicas, manteniendo vigente la necesidad de disponer de modelos teóricos, derivados de la investigación empírica, apropiados para la comprensión y sistematización del enfoque, que permitan su transferencia a la formación docente.

En síntesis, los énfasis del lustro se encuentran en: a) la comparación de efectos de mayor o menor andamiaje, estructuración o mediación del formador en la experiencia reflexiva del estudiante, b) mayor rigurosidad científica, con una creciente incorporación de investigaciones que evalúan línea base y resultados al finalizar las experiencias, a fin de establecer diferencias.

El avance en el campo deriva en un giro considerable capaz de tensionar la definición de reflexión como producto social que requiere mediación o andamiaje de otro y de herramientas que la sitúen (GUERRA, 2009), particularmente poniendo en suspenso el rol de la mediación en dicha construcción; y sugiriendo más bien la necesidad de resituar el rol del conocimiento teórico y técnico en el desarrollo de la reflexión docente, en tanto insumo a la luz del cual se posibilita dicha reflexión, bajo la forma de fundamentación teórica de las decisiones pedagógicas.

De manera similar, son tensionadas las condiciones mínimas para el desarrollo reflexivo propuestas por la misma autora: a) tiempo y espacio para la reflexión, que bien podría entenderse como una situación creada por una cultura que no prioriza la reflexión (THOMPSON; PASCAL, 2012), y b) formadores preparados para propiciar la reflexión, que podrían ser menos gravitantes de prosperar la investigación que sostiene que un desarrollo reflexivo persistente y profundo se asocia a la oportunidad extendida en el tiempo de reflexión libre, no estructurada, mediada por herramientas que probablemente incorporan andamios intrínsecos como los videos o animaciones.

Simultáneamente, aparece la necesidad de considerar nuevos elementos, factores o variables capaces de impactar el desarrollo reflexivo, que hasta el momento parecen no encontrarse definidos en las comprensiones del fenómeno, como por ejemplo, los de carácter sociopolítico; a la vez que se observa la necesidad de trascender a la investigación sobre experiencias aisladas y de profundizar en investigación orientada a captar factores críticos para el desarrollo reflexivo y formas en que la reflexión acerca de la experiencia se transforman en conocimiento profesional y modifican la práctica.

\section{Referencias}

ALPER, Ayfer. Critical Thinking Disposition of Pre-Service Teachers. Egitim Ve Bilim-Education and Science, Ankara, v. 35, n. 158, p. 14-27, 2010.

BATES, Alisa; RAMÍREZ, Laurie; DRITS, Dina. Connecting university supervision and critical reflection: mentoring and modeling. The Teacher Educator, Muncie, v. 44, p. 90-112, 2009.

BERLINER, David. In pusuit of the expert pedagogue. Educational Researcher, Washington, v. 15, p. 5-13, 1986.

BLOMBERG, Geraldine et al. Understanding video as a tool for teacher education: investigating instructional strategies to promote reflection. Instructional Science, Cham, v. 42, n.3, p. 443-463, 2014. 
CHUNG, Huy; VAN ES, Elizabeth. Pre-service teachers' use of tools to systematically analyze teaching and learning. Teachers and Teaching, Nottingham, v. 20, n. 2, p. 113-135, 2014

CONCHA, Soledad et al. Reflexión pedagógica en base a casos y dominio de lenguaje académico en estudiantes de cuarto año de pedagogía en educación básica. Calidad en la Educación, Santiago de Chile, n. 38, p. 81-113, 2013.

CORNEJO, José. El pensamiento reflexivo entre profesores. Pensamiento Educativo, Santiago de Chile, v. 32, p. 343-373, 2003.

CRUICKSHANK, Donald. Reflective teaching: the preparation of students of teaching. Reston: Association of Teacher Educators, 1987.

DANIEL, Graham; AUHL, Greg; HASTINGS, Wendy. Collaborative feedback and reflection for professional growth: preparing firstyear pre-service teachers for participation in the community of practice. Asia-Pacific Journal of Teacher Education, v. 41, n.2, 159-172, 2013.

DARLING-HAMMOND, Linda; BRANSFORD, John. Preparing teachers for a changing World: what teachers should learn and be able to do. San Francisco: Jossey-Bass, 2005.

DEWEY, John. Cómo pensamos: nueva exposición de la relación entre pensamiento reflexivo y proceso educativo. Barcelona: Paidós, 1989

FENDLER, Lynn. Teacher reflection in a hall of mirrors: historical influences and political reverberations. Educational Researcher, Washington, v. 32, n. 3, p. 16-25, 2003.

FRICK, Liezel; CARL, Arend;BEETS, Peter. Reflection as learning about the self in context: mentoring as catalyst for reflective development in pre-service teachers. South African Journal of Education, Pretoria, v. 30, n. 3, p. 421-437, 2010.

GELFUSO, Andrea; DENNIS, Danielle. Getting reflection off the page: The challenges of developing support structures for preservice teacher reflection. Teaching and Teacher Education, v. 38, p. 1-11, 2014.

GUERRA, Paula. Revisión de experiencia de reflexión en la formación inicial de docentes. Estudios Pedagógicos, Valdivia, v. 35, n. 2, p. 243-260, 2009.

GUROL, Aysun. Determining the reflective thinking skills of pre-service teachers in learning and teaching process. Energy Education Science and Technology Part B-Social and Educational Studies, Trabzon, v. 3, n. 3, p. 387-402, 2011.

HATTON, Neville; SMITH, David. Reflection in teacher education: towards definition and research into the use of diaries and journal to facilitate reflection. Teacher in Higher Education, v. 2, n. 2, p. 103-121, 1995.

HOLLOWAY, Susan; GOUTHRO, Patricia. Teaching resistant novice educators to be critically reflective. Discourse, v. 32, n. 1, p. 29-41, 2011.

JAY, Joelle; JOHNSON, Kerry. Capturing complexity: a typology of reflective practice for teacher education. Teaching and Teacher Education, v. 18, n. 1, p. 73-85, 2002.

JONES, Mellita; RYAN, Josephine. Learning in the practicum: engaging pre-service teachers in reflective practice in the online space. Asia-Pacific Journal of Teacher Education, v. 42, n. 2, p. 132-146, 2014.

KAASILA, Raimo; LAURIALA, Anneli. How do pre-service teachers' reflective processes differ in relation to different contexts?. European Journal of Teacher Education, v. 35, n. 1, p. 77-89, 2012.

MARCOS, Juanjo; SANCHEZ, Emilia; TILLEMA, Harm. Promoting teacher reflection: what is said to be done. Journal of Education for Teaching, v. 37, n. 1, p. 21-36, 2011.

MOORE-RUSSO, Deborah; WILSEY, Jillian. Delving into the meaning of productive reflection: a study of future teachers' reflections on representations of teaching. Teaching and Teacher Education, v. 37, p. 76-90, 2014.

OAKLEY, Grace; PEGRUM, Mark; JOHNSTON, Shannon. Introducing e-portfolios to pre-service teachers as tools for reflection and growth: lessons learnt. Asia-Pacific Journal of Teacher Education, v. 42, n. 1, p. 36-50, 2014. 
OVENS, Alan; TINNING, Richard. Reflection as situated practice: A memory-work study of lived experience in teacher education. Teaching and Teacher Education, v. 25, n. 8, p. 1125-1131, 2009.

ROSS, Jen. Performing the reflective self: audience awareness in high-stakes reflection. Studies in Higher Education, v. 39, n. 2, p. 219-232, 2014.

RUSSELL, Tom. Cambios paradigmáticos en la formación de profesores: Peligros, trampas y la promesa no cumplida del profesional reflexivo. Encounters on Education, Ottawa, v. 13, p. 71-91, 2012.

RUSSELL, Tom. Can reflective practice be taught? Reflective Practice, v. 6, n. 2, p. 199-204, 2005.

SCHÖN, Donald. El profesional reflexivo: cómo piensan los profesionales cuando actúan. Barcelona: Paidós, 1998.

SCHÖN, Donald. La formación de profesionales reflexivos: hacia un nuevo diseño de la enseñanza y el aprendizaje en las profesiones. Madrid: Paidós, 2010.

SCHÖN, Donald. The reflective practitioner: how professionals think in action. New York: Basic Books, 1983.

SILVA, Alejandra et al. Modelo para el desarrollo de capacidades reflexivas y didácticas en estudiantes de educación parvularia y básica durante la práctica profesional. Santiago, Chile: Universidad Metropolitana de Ciencias de la Educación, 2012. Proyecto Fonide: F-611145.

SIMS, Linda;WALSH, Daniel. Lesson Study with preservice teachers: Lessons from lessons. Teaching and Teacher Education, v. 25, n. 5, p. $724-733,2009$.

THOMPSON, Neil; PASCAL, Jen. Developing critically reflective practice. Reflective Practice, v. 13, n. 2, p. 311-325, 2012.

VAN - MANEN, Max. Linking ways of knowing with ways of being practical. Curriculum Inquiry, Toronto, v. 6, n. 3, p. 205-228, 1977.

YAFFE, Elika. The reflective begginer: using Theory and practice to facilitate reflection among newly qualified teachers. Reflective Practice, v. 11, n. 3, p. 381-391, 2010.

YOUNGS, Peter; BIRD, Tom. Using embedded assessments to promote pedagogial reasoning amonh secondary teaching candidates. Teaching and Teacher Education, v. 26, n. 2, p. 185-198, 2010.

ZEICHNER, Kenneth. El maestro como profesional reflexivo. Cuadernos de Pedagogía, v. 220, p. 44-49, 1993.

ZHU, Xihe. Student teachers' reflection during practicum: plenty on action, few in action. Reflective Practice, v. 12, n. 6, p. 763775, 2011.

ZINK, Robyn; DYSON, Michael. What does it mean when they don't seem to learn from experience?. Cambridge Journal of Education, Cambridge, v. 39, n. 2, p. 163-174, 2009.

Recibido en: 15.01.2016

Aprobado en: 13.09.2016

Andrea Ruffinelli investiga en torno a la formación inicial docente, iniciación profesional y formación de docentes reflexivos en la Universidad Alberto Hurtado, Santiago, Chile. Es (c) doctora candidata en Ciencias de la Educación, Universidad Católica de Chile, magister en Educación Especial y en Educación de la misma universidad. 